\title{
Contribution of instructions to the effects of two types of symbolic reinforcers on the discrimination learning of children
}

\author{
JANET TAYLOR SPENCE, JAMES \\ ARMSTRONG, and ROBERT CONRAD, \\ University of Texas at Austin, Austin, Tex. \\ 78712
}

The study investigated the effects on discrimination learning of two reinforcers ("right" or a buzzer) under instructional conditions in which $S$ s received either a full explanation of the reinforcement procedures, partial explanation (no mention of reinforcer omission, blank), or no explanation. Noninformed $S$ s were significantly poorer than the fully informed. In the right group, this inferiority was due to the relative ineffectiveness of blank as a negative reinforcer or misinterpretation of blank's meaning, and in the buzzer group, primarily to the ineffectiveness of the buzzer as a positive reinforcer. The partially informed $S$ s were more similar in performance to the fully informed than the noninformed.

In a recent study, Cairns (1967) found no significant difference between children's performance on a concept-identification task under two types of symbolic reward, a buzzer and the word "right." However, groups given prior instructions about the reinforcement procedures performed markedly better than those given no information about the reinforcers, results interpreted as indicating that under noninformative instructional conditions, not only an informationally ambiguous event such as a buzzer but also "right" is an ineffective reinforcer.

This explanation is based on an unstated assumption that is frequently made implicitly in studies of this type, namely, that the failure of an overt reinforcer to occur (blank) had identical (or in the case of positive vs negative reinforcement comparisons, symmetrical) effects on the performance of the groups being compared. In some instances, the assumption may be untenable. For example, under conditions in which Ss are uninstructed about the reinforcers, groups reinforced by "right" are typically poorer in performance than those reinforced by "wrong" (e.g., Curry, 1960; Buss \& Buss, 1956). Studies by the present writer (e.g., Spence, 1966), utilizing a two-alternative discrimination task, suggest that the performance inferiority of the right group comes about because of the tendency of some Ss to react to blank as if it were "right." This misinterpretation of blank is evidenced in performance by the use of deviant response strategies, typically repetition of all previous responses, correct and incorrect.

In the Cairns study described above, it seems quite possible that for uninformed Ss, "right" was neither an ineffectual nor an ambiguous reinforcer; the inferior performance of this group, in comparison to Ss informed about the reinforcers, may instead have been due to their reactions to blank, some individuals misinterpreting it and still others extracting little or no information from it. Uninformed $S s$ in the buzzer condition, while perhaps less likely to misinterpret blank, may have found both the buzzer and blank informationally ambiguous. These possibilities were tested in the present study by duplicating the experimental conditions of the Cairns study, but utilizing a verbal-discrimination task which permitted separate assessment of the effects on performance of the overt reinforcers and of blank. A third instructional group was also tested in which Ss were informed about the overt reinforcers ("right" or the buzzer), but not about the information value of blank.

\section{METHOD}

The Ss were 64 boys and 64 girls enrolled in the third and fourth grades of public schools in Austin, Texas. The verbal-discrimination list consisted of 12 pairs of unrelated but familiar words. Four serial orders of the list were prepared, the right-left order of each pair being counterbalanced across trials.

Each $S$ was told that one member of each pair was "right" and the other "wrong," his task being to learn to choose the "right" word. The fully-informed-right (F-R) and fully-informed-buzzer (F-B) groups were instructed both about the consequence of correct responses ("right" or buzzer) and of incorrect responses (blank). Partially-informed groups (P.R and P-B) were instructed only about the overt reinforcers, while the two noninformed groups (N-R and N-B) were merely told that they would find out as they went along when they had responded correctly. After administration of a two-pair practice list, the experimental list was given for 16 trials or until a criterion of two successive perfect trials was reached, whichever occurred first.
On the first (guessing) trial, the $S$ 's responses to half of the pairs, as designated by a prearranged schedule were specified as "correct" on this and on all subsequent trials, and his responses to the other half as "incorrect." For each pair, the overt reinforcer was delivered for correct responses immediately after S's choice, and the next pair exposed approximately $1 / 2 \mathrm{sec}$ later.

\section{RESULTS AND DISCUSSION}

Since a substantial number of Ss mastered the list well before the 15th learning trial, only the results from the first 10 trials were analyzed. Comparison of the means of the total number of correct responses per $S$ reveals that the two types of reinforcers produced equivalent performance in the fully-informed conditions ( $F-R=91.25$, $F-B=90.83$ ) and in the noninformed conditions $(\mathrm{N}-\mathrm{R}=73.04, \quad \mathrm{~N}-\mathrm{B}=76.17)$, although the latter groups were markedly inferior to the fully-informed Ss. In the partially-informed condition, however, the two reinforcement groups differed, the performance of those reinforced by "right" being intermediate to that of the other two instructional conditions $(\overline{\mathrm{X}}=80.79)$, and those reinforced by the buzzer doing as well as the fully-informed Ss ( $\bar{X}=91.63)$. An analysis of variance of these data indicated significant terms for Instructions $\bar{F}=10.93, \mathrm{df}=2 / 138, \mathrm{p}<.01)$ and for the Reinforcer by Instructions interaction $(\mathrm{F}=3.35, \mathrm{df}=2 / 138, \mathrm{p}<.05)$.

Two further analyses were performed to determine the factors contributing to these performance differences. First, each record was examined for the presence of deviant response strategies. Thirteen of the $128 \mathrm{Ss}$ were identified as exhibiting a repetition strategy in which all previous responses tend to be repeated on the subsequent trial. (The criterion for identifying these "repeaters" was six or more trials on which all incorrect responses of the previous trial were prefectly repeated; all other Ss scored three or less on this measure, with a mode of zero.) As anticipated, most of these 13 "repeaters" were in the groups reinforced by "right" and not fully informed about the reinforcement procedures (eight and two Ss in the N-R and $P-R$ groups, respectively). The remaining three "repeaters" were in the N-B group.

In the second analysis, the number of correct responses on each trial was determined for each $S$ and then for the percentages of these responses that continued to be correct on the following trial. The percentage of correct responses that had been incorrect on each previous trial was also determined in a similar manner. These data are plotted in Fig. 1.

Inspection of the figure indicates that, in the noninformed-right condition, "right" was as effective in producing repetition of correct responses as it was in the other two 


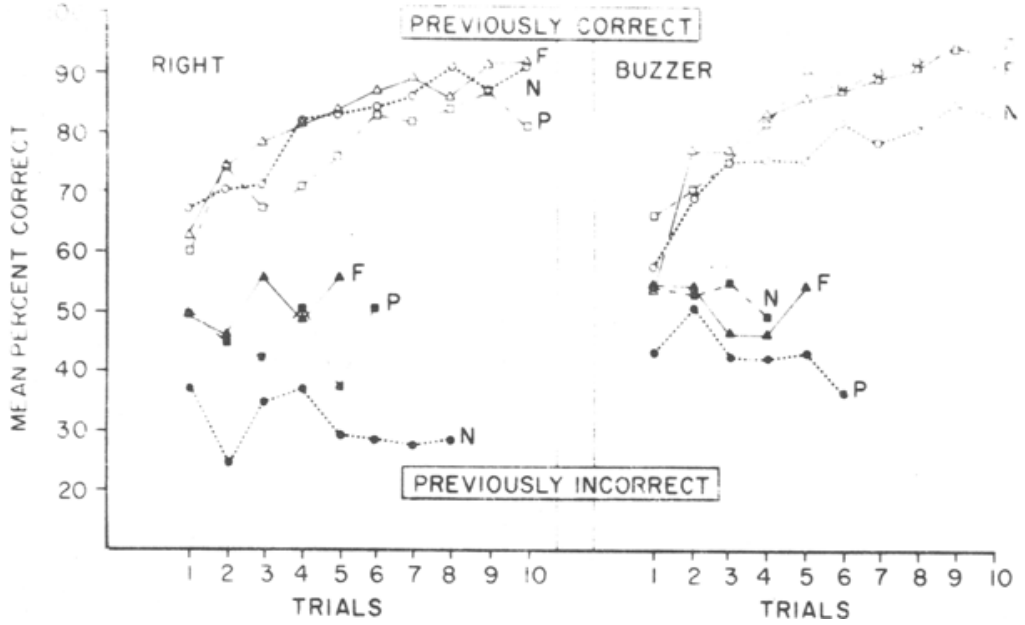

instructional conditions, but that blank following incorrect responses was less effective in this group in producing a shif to correct responses. The presence of the eight "repeaters" in the N-R condition contributed heavily to the latter result However, when the records of all "repeaters" were eliminated, the curve of per cent correct following incorrect for the N-R group rose, but was still markedly lower than that of the other two instructional groups. Thus, the performance inferiority of the noninformed Ss reinforced by "right" appears to be due to the relative ineffectiveness of blank, rather than of the overt reinforcer, some Ss reacting to blank as if it were "right" ("repeaters") and others as if it were informationally ambiguous or meaningless.

In the noninformed-buzzer condition, blank was also less effective than it was in the other buzzer conditions. However, the discrepancy was less marked than in the case of the groups reinforced by "right," primarily because of the smaller number of "repeaters" in the N-B group. The major difference between the N-R and N-B conditions, however, concerned the effects of the overt reinforcer. While "right" was as effective a positive reinforcer in the N-R group as in the other groups reinforced by "right," the buzzer in the N-B group was less effective a positive reinforcer than it was in the F-B and P-B conditions in which Ss had been given an explanation of its meaning. Only one S, however, appeared to "misinterpret" the buzzer, i.e., re acting to it as if it signaled incorrect responses by systematically coming to select more and more responses followed by blank.

The behavior of $S s$ in the partially-informed buzzer group was in all respects indistinguishable from that of the fully-informed group. The P-R group, however, did not perform as well as the F-R, a result only partly explicable by the presence of two "repeaters" among the P-R Ss. As may be seen in Fig. 1, blank was somewhat less effective as a negative reinforcer than in the $F \cdot R$ group, and "right" was less effective as a positive reinforcer than in either the F-R or N-R groups. The latter results, in particular, suggest that the poorer performance of the P-R Ss in comparison to F-R may partially be the result of sampling error. It would, none theless, seem wise in studies in which it is desirable for Ss to understand the reinforcement procedures to explain the
EDITH D. NEIMARK and MARCIA HORN, Douglass College, Rutgers, The State University, New Brunswick, N.J. 08903

Data on performance of a 2-year-old girl on a series of fixed-trial discrimination and oddity problems are presented. There is evidence of the development of a discrimination-learning set, and, possibly, of an oddity set as well. After reviewing existing evidence, it is suggested that 24-30 months may constitute a lower limit for the attainment of learning set in humans.

Harlow, Harlow, Rueping, \& Mason (1960) have presented evidence that learning-set formation in the young rhesus is a function of age rather than of prior training, and that young monkeys below 90 days of age do not acquire a learning set. Comparison of the available evidence on learning set in children suggests a similar conclusion. Although Levinson \& Reese (1967) found no correlation between CA and trials to criterion for their 36- to 66-month-old Ss, Koch \& Meyer (1959) reported a correlation of -.59 between MA and trials to criterion for children 29-66 months old Haves. Thompson, and Hayes (1953) report data for six children: the two
Fig. 1. Mean per cent correct on given trial for previously correct and incorrect responses for the three instructional groups in the right and buzzer conditions. (Analyses of incorrect response discontinued when one or more Ss reached criterion of perfect performance.)

informational characteristics of not only the overt reinforcers but also of blank.

\section{REFERENCES}

BUSS, A. H., \& BUSS, E. H. The effect of verbal reinforcement combinations on conceptual learning. Journal of Experimental Psychology. 1956, 52, 283-287.

CAIRNS, R. B. Informational properties of verbal and nonverbal events. Journal of Personality \& Social Psychology, 1967, 5, 35 3-357.

CURRY. C. The effects of verbal reinforcement combinations on kearning in children. Journal of Experimental Psychology, 1960, 59,434.

SPENCE. J.T. Verbal discrimination performance as a function of instructions and verbal reinforcement combinations in normal and retarded children. Child Development. 1966. $37,269-281$

\section{NOTE}

1. The investigation was supported, in part, by Grant HD01980 from the National Institute of Mental Health.

\section{Development of discrimination and oddity learning set in a two-year-old girl}

6-year-olds and a bright 4year-old learned rapidly, a normal 4-year-old learned more slowly, and the two 2-year-olds gave no evidence of acquiring a learning set at all. Thus, there is some question about whether or not a child of $2-21 / 2$ years can develop a discrimination leaming set. The present experiment attempts to answer that question and, in addition, to provide evidence on performance on oddity problems by the 2-year-old. A review of the literature showed there is no evidence of oddity learning in children below 4

\section{SUBJECT}

The sole $S$, daughter of the second author, was tested in her own home over a 3-month period. She was 26 months old at the start of training and was already speaking in sentences. She could accurately discriminate and name colors -at least to the extent of correct response to a request to select all the blue (red, etc.) from an array of colored cards. By the same criterion she could also discriminate squares from triangles (although she did not initially know the word "triangle," she learned it as a result of this test and used it spontaneously later).

$$
\text { APPARATUS }
$$

A small wooden tray, $12 \times 2 \frac{1}{2}$ in.. with 